\title{
DyTER - Dynamic Track and Event Reconstruction
}

\author{
Michael Papenbrock ${ }^{1, *}$ on behalf of the PANDA collaboration \\ ${ }^{1}$ Uppsala University, Department for Physics and Astronomy
}

\begin{abstract}
The future PANDA experiment at FAIR experiment aims to cover a wide range of processes in antiproton-proton collisions at event rates of up to $20 \mathrm{MHz}$. Such event rates make reconstruction a challenging task for the purely software-based event filter. Investigating complex event topologies with displaced vertices increases the difficulty even further. Here we present two attempts to meet these future challenges: an algorithm for track reconstruction based on pattern matching with pre-determined look-up tables, and as a continuation of this approach a system of neural networks for identifying specific particle track candidates and predicting their momentum.
\end{abstract}

\section{Track and Event Reconstruction at PANDA}

PANDA (Antiproton Annihilation at Darmstadt) [1] is a multi-purpose detector (see figure 1) currently under construction at the future Facility for Antiproton and Ion Research (FAIR) in Darmstadt, Germany. The fixed-target experiment aims to investigate a wide range of antiproton induced reactions with an angular coverage of nearly $4 \pi$. The High Energy Storage Ring (HESR) will deliver high intensity antiproton beams with momenta of up to $15 \mathrm{GeV} / \mathrm{c}$ and a nearly continuous beam structure. The event rates are expected to reach up to $20 \mathrm{MHz}$. Such event rates make online reconstruction a challenging task for the purely software-based event filter.

Amongst the many processes PANDA will study, there are those with challenging topologies involving secondary vertices. Considering that in many algorithms the interaction point often serves as a powerful constraint, reconstructing tracks that originate several centimetres or even metres away from the primary vertex can drastically increase the complexity of the algorithms. Therefore, matching track candidates with possible vertices may increase the computing requirements dramatically. Hence, flexible algorithms are needed for track and event reconstruction.

Here, hyperon reactions prove to be good benchmark channels due to their complex topologies. These include displaced vertices, intersecting tracks, and final state particles which reach all of PANDA's subdetectors for charged particle detection. The following studies put a particular focus on the $\bar{p} p \rightarrow \bar{\Lambda} \Lambda$ reaction and the Straw Tube Tracker (STT) subdetector.

\section{Pattern Matching}

One approach to track reconstruction can be found in the form of pattern matching. The basic concept is to simulate virtually every track that can occur in the detector in a real measurement

\footnotetext{
*e-mail: michael.papenbrock@physics.uu.se
} 


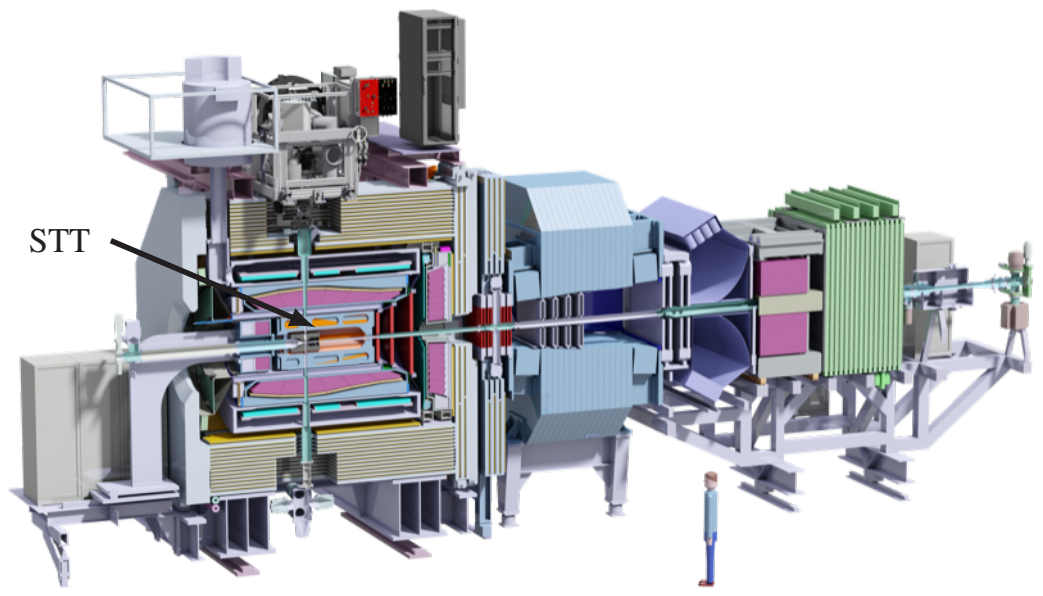

Figure 1. The PANDA detector. Indicated is the Straw Tube Tracker (STT).

and store its distinct combination of activated detector elements, the pattern, in some form of look-up table. For reconstruction, the incoming detector signatures are compared with the recorded patterns and the best matches are identified as possible track candidates. This approach is appealing as it has proven to be rather reliable and robust over the years [2]. Furthermore, it lends itself well to implementation on hardware accelerators such as FPGAs, which is of particular importance when considering it for potential online use, i.e. while the experiment is running and acquiring data.

The Straw Tube Tracker of PANDA is divided into six individual sectors which are read out separately, each with their own electronics [3]. When creating the look-up table for the pattern matching algorithm, patterns are generated for each sector. Therefore, a single track can create multiple patterns if it crosses sectors (see figure 2). Training data are generated with PandaRoot [4], from which patterns are extracted. First, events are created with an appropriate event generator. The resulting tracks are then propagated through the detector using Geant4. After a digitisation stage which creates realistic hit data, track candidates are identified by an ideal track finder using Monte Carlo truth information. These steps yield all the information needed to create a pattern database. A pattern is composed as follows: The actual hit pattern consisting of tube identifiers (tube IDs), the momentum of the particle creating that pattern, the time stamps of the hits, an identifier for the sector (sector ID), as well as the number of pattern occurrences with the same set of tube IDs.

Considering only trajectories from delayed decays, as is the case in hyperon production reactions, the range of possible combinations of corresponding activated straw tubes becomes rather large. In order to estimate the feasibility of the pattern matching approach for such reactions, the amount of needed training data and eventual size of the pattern database have to be considered. For a first estimate, we generated one million events of the $\bar{p} p \rightarrow \bar{\Lambda} \Lambda$ reaction, yielding $\approx 2.2$ million patterns. Afterwards, patterns with an identical set of tube IDs were merged into one entry. The merged entry contained the shared hit pattern and sector ID, the number of occurrences of this pattern, and a list of momenta and time stamps from the original entries. While a subset of patterns showed a high repetition rate, up to $O(1000)$ occurrences per pattern, a very large set of generated tracks would be necessary before no more new, unique patterns appear, as illustrated in figure 3 (black dots). However, only considering exact matches is a steep requirement. It is reasonable to assume that two tracks are very 


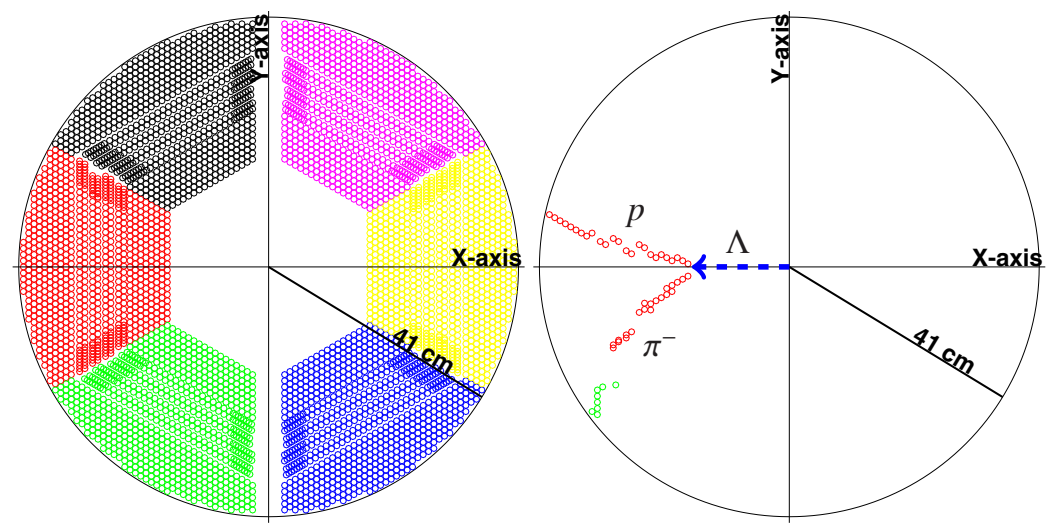

Figure 2. Left: A sketch of the PANDA Straw Tube Tracker divided into six sectors. Right: The signature of a $\bar{p} p \rightarrow \bar{\Lambda} \Lambda$ event. The $\Lambda$ traverses into the STT and decays into a $p$ and a $\pi^{-}$, creating hit patterns across multiple sectors.

similar, or effectively the same, if they share a large fraction of tube IDs in their pattern. As a first estimate, we merged entries that shared $90 \%$ of their tube IDs. These are also shown in figure 3 (blue triangles). The number of entries, i.e. merged patterns, is significantly reduced to $\lesssim 100000$, which corresponds to a database size of less than $100 \mathrm{MB}$. Further investigation will aim to find a good balance between accuracy and computing requirements.

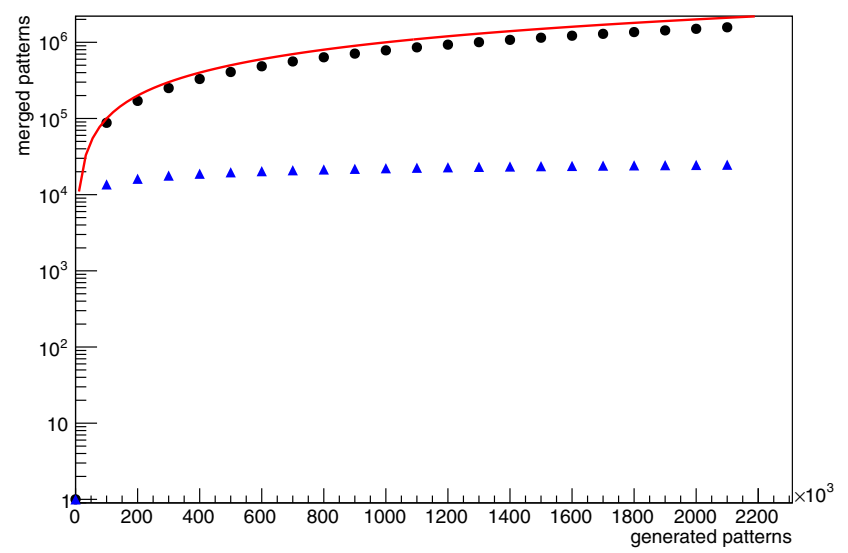

Figure 3. The merged patterns plotted versus the generated ones for $1000000 \bar{p} p \rightarrow \bar{\Lambda} \Lambda$ events. The red line illustrates the case when no merging is done at all. The black points represents the number of patterns in the database after those with identical sets of tube IDs have been merged. The blue triangles show the number of patterns after those with $90 \%$ matching tube IDs have been merged.

While this can be further developed into a standalone track finder suitable to be run on FPGAs, we also explored the possibility to the generated database as an input for machine learning algorithms. 


\section{Neural networks}

In recent years, the field of machine learning and artificial intelligence has seen an incredible rise in popularity. The applications and implementations cover a wide range, with image processing using convolutional neural networks (CNN) among the more common ones [5]. While neural networks had a presence in high energy physics for many decades, this recent surge has not gone unnoticed in this realm either. In fact, it has lead to considering machine learning algorithms for applications ranging from track reconstruction to high level analysis [6]. The situation seems more appealing than ever. For example, neural networks can be efficiently mapped onto the hardware of an FPGA. Furthermore, the manufacturers like nVidia have acknowledged the trends and are including tensor processing cores designed for machine learning algorithms on their current GPUs. This allows these techniques to achieve performances that would be otherwise difficult to reach with more conventional algorithms.

As an extension of the developments outlined in section 2 we explored the possibilities of employing a neural network for the purpose of particle tracking with the PANDA detector. As before, we focused on the Straw Tube Tracker. A first prototype was developed [7] with a simplified problem description:

- Can we identify one specific particle track within a $\bar{\Lambda} \Lambda$ event topology?

- Can we extract physical observables, i.e. the transversal momentum components?

To answer these questions, two fully connected neural networks were implemented, one for pattern recognition, the other for momentum regression. The implementation was done in MATLAB [8] and the networks were trained with data from the aforementioned pattern matching algorithm.

The purpose of the pattern recognition is to identify a proton track candidate in the events of a $\bar{p} p \rightarrow \bar{\Lambda} \Lambda$ test sample. The input layer consists of the activated tube IDs of all raw Straw Tube Tracker hits in an event. The output layer then returns the set of tube IDs for the specific particle track. In between, there are four hidden layers. The features of the network are given in table 1.

\begin{tabular}{c|c|c} 
Layer & Number of neurons & Activation function \\
\hline Input layer & 4542 & - \\
Hidden layer 1 & 300 & ReLU \\
Hidden layer 2 & 200 & ReLU \\
Hidden layer 3 & 200 & ReLU \\
Hidden layer 4 & 300 & ReLU \\
Output layer & 4542 & Sigmoid
\end{tabular}

Table 1. Properties of the neural network for pattern recognition. The used activation functions are the rectified linear unit activation function $(\operatorname{ReLU})$ with $f(x)=\max (0, x)$ in $[0, \infty)$ and the Sigmoid function with $f(x)=\frac{1}{1+e^{-x}}$ in $(0,1)$. Taken from [7].

The output of the pattern recognition network then serves as input for the momentum regression network with the goal to obtain an estimate for the track's $p_{x}$ and $p_{y}$ momentum components, which constitutes the output layer. Six hidden layers are used. More features for this network can be found in table 2 .

A data set containing $\approx 750000 \bar{p} p \rightarrow \bar{\Lambda} \Lambda$ events was simulated using PandaRoot. The training algorithm used the Adam optimisation [9], for which batch training is commonly used. Hence, the networks were trained with over 2000 batches from this training set with 


\begin{tabular}{c|c|c} 
Layer & Number of neurons & Activation function \\
\hline Input layer & 4542 & - \\
Hidden layer 1 & 400 & ReLU \\
Hidden layer 2 & 300 & ReLU \\
Hidden layer 3 & 200 & ReLU \\
Hidden layer 4 & 100 & ReLU \\
Hidden layer 5 & 50 & ReLU \\
Hidden layer 6 & 30 & ReLU \\
Output layer & 2 & Identity
\end{tabular}

Table 2. Properties of the neural network for momentum regression. The used activation functions are the rectified linear unit activation function (ReLU) with $f(x)=\max (0, x)$ in $[0, \infty$ and the Identity function with $f(x)=x$ in $(-\infty, \infty)$. Taken from [7].

one batch containing 1000 events. A separate set containing 10000 events was used for validation.

The workflow of the neural networks is visualised in figure 4. A state vector derived from the raw hits of the Straw Tube Tracker is used as an input layer. The size of 4542 is the total number of tubes in the detector. Then, the networks determines a probability for each tube in the detector for a proton having been present, retaining only those tubes which yielded a proton hit probability larger than 0.99. This output is correlated with the actual input, keeping only those tubes which gave a physical hit signal. The resulting state vector is passed as input to the momentum regression network which gives an estimate for $p_{x}$ and $p_{y}$. This is compared here with the true Monte Carlo momentum that was originally generated.

The left part of figure 5 shows prediction accuracy of the pattern recognition network. It reaches a maximum accuracy of approximately 95\%. Furthermore, with the performance on the test data being very similar to that on the training data, signs of overfitting are rather small. The same plot for the momentum regression network can be seen in the right hand part of figure 5. A prediction was defined as correct if the momentum vector falls within $5^{\circ}$ of the Monte Carlo truth momentum vector and within $10 \%$ of its magnitude. Here, the accuracy is notably lower and for larger batch numbers, signs of overfitting increase. The lower accuracy could originate from the fact that only the tube IDs were used as input for the neural network, but not the isochrone radii. Incorporating this information in the future might lead to a better result. Considering that the momenta are meant to be used for online event reconstruction, an accuracy of $\gtrsim 95 \%$ is desired.

In order to evaluate the performance of this prototype from the perspective of a track finding algorithm, we define the following quantities

$$
\begin{aligned}
\text { prediction accuracy } & =\frac{T H+T M}{T H+T M+F H+F M}, \\
\text { efficiency } & =\frac{T H}{T H+F M}, \\
\text { purity } & =\frac{T H}{T H+F H},
\end{aligned}
$$

with $T H$ (true hits) being the number of correctly predicted hits in the STT, $T M$ (true misses) correctly predicted misses, $F H$ (false hits) falsely predicted hits, and $F M$ (false misses) falsely predicted misses. These are plotted in figure 6 against the threshold value for the aforementioned probability cut. These give insight how the threshold value can be optimised 


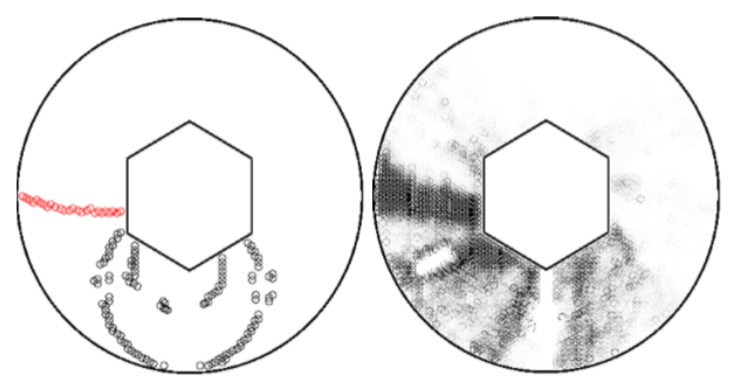

(a)

(b)

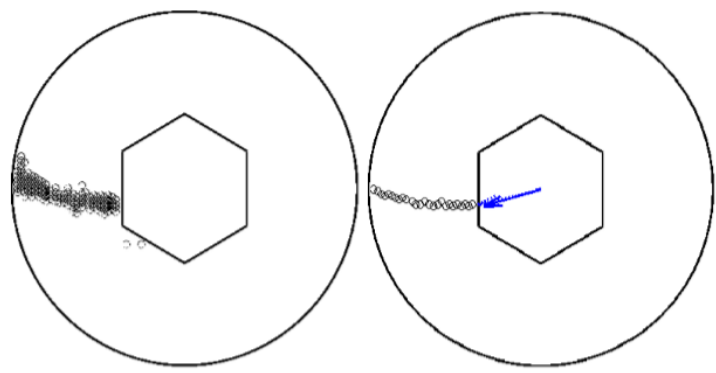

(c)

(d)

Figure 4. Visualisation of the neural networks. (a) The input for the pattern recognition network. The proton track is highlighted in red for the reader. (b) The raw output of the network. The intensity corresponds to the probability that a proton was present. (c) The output of the network after after a threshold cut was applied for the probability. Only probabilities $>0.99$ were retained. (d) The filtered hits after correlation with the initial input. Also shown are the predicted momentum (solid arrow) together with the true momentum (dotted arrow).
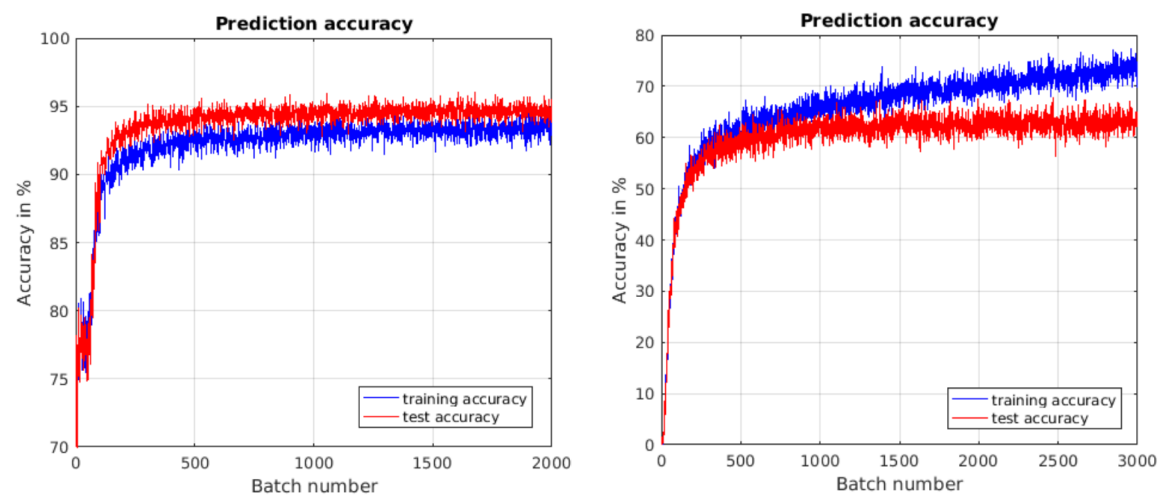

Figure 5. The prediction accuracy of the pattern recognition network (left) and the momentum regression network (right) as a function of the number of batches used in training. Taken from [7]. 

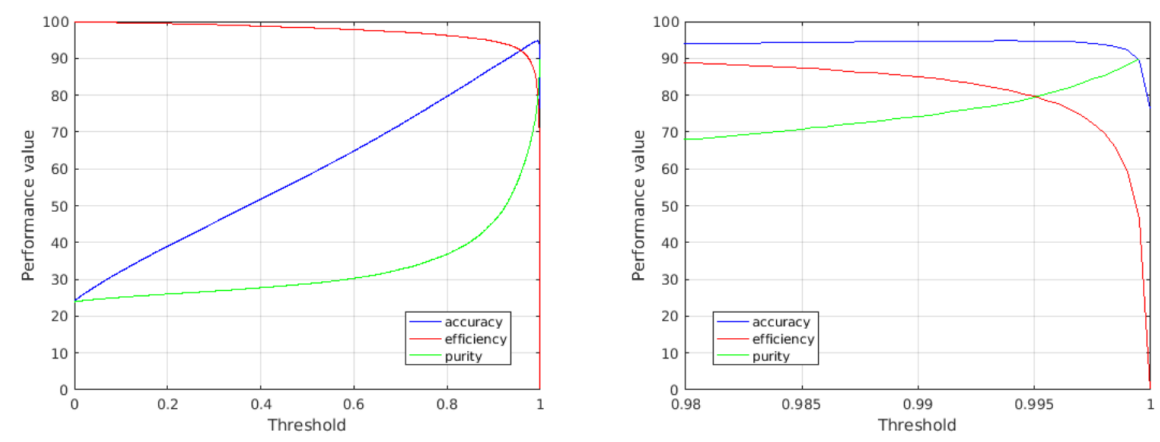

Figure 6. The prediction accuracy, efficiency, and purity for the pattern recognition network as a function of the threshold value for the full range (left) and the final $2 \%$ (right). Taken from [7].

to find a suitable compromise between accuracy, efficiency, and purity, in particular in the range between 0.98 and 1.0.

\section{Conclusions}

Developing track and event reconstruction for the PANDA experiment is a challenging task. The high expected event rates coupled with a purely software-based triggering system puts high demands on the reconstruction algorithms, in particular when being used online. A pattern matching approach has been studied, which uses pre-determined look-up tables in order to find expected track signatures in the incoming data stream.

A natural extension of this concept is the exploration of machine learning techniques for track and event reconstruction, in particular with neural networks. A first prototype was developed showing promising results in the areas of specific track identification and momentum regression. These networks tend to be highly parallelisable, making them a good match for GPUs or FPGAs. Hence, they may present a good candidate to meet the performance requirements in high event rate scenarios.

With our future efforts we will explore the applicability of both the pattern matching and neural network algorithms to be run on FPGAs. Furthermore, the neural network solutions are currently being ported to Python/TensorFlow and being extended to all types of tracks and other tracking detectors in PANDA.

The authors are grateful to the Knut and Alice Wallenberg Foundation for the support.

\section{References}

[1] PANDA Collaboration, arXiv:0903.3905 (2009)

[2] N. Ilic, JINST 12, C02052, (2017)

[3] PANDA Collaboration, Eur.Phys.J. A49, 25 (2013)

[4] S. Spataro, JoP: Conf. Series 119, 032035 (2008)

[5] Y. Bengio, Y. LeCun, G. Hinton, Nature 521, 436-44, (2015)

[6] K. Albertsson et al., arXiv:1807.02876, (2018)

[7] A. Jonnarth, A. Hedkvist, project report, (2018), http://www.it.uu.se/edu/course/ homepage/projektTDB/ht17/project01/Project01b_report.pdf

[8] MATLAB, The MathWorks, Inc., Natick, Massachusetts, United States.

[9] D.P. Kingma, J.L. Ba, arXiv:1412.6980, (2014) 